\title{
Signal Conditioning of Carbon Nanotube Thin Film Loudspeakers
}

Asha Hall ${ }^{1}$, Member IEEE, Jeremy Gaston ${ }^{2}$, Wosen Wolde ${ }^{1}$, Edwards Baker ${ }^{3}$, Morihiro Okada ${ }^{3}$, YuHuang Wang ${ }^{3}$, Shashi Karna, ${ }^{4}$ Senior Member IEEE

${ }^{1}$ US Army Research Laboratory, Vehicle Technology Directorate, Aberdeen Proving Ground, MD 21005

${ }^{2}$ US Army Research Laboratory, Human Research Engineering Directorate, Aberdeen Proving Ground, MD 21005

${ }^{3}$ University of Maryland, Department of Chemistry and Biochemistry, College Park, MD, 20742

${ }^{4}$ US Army Research Laboratory, Weapons and Materials Research Directorate, Aberdeen Proving Ground, MD 21005 Email:asha.j.hall.civ@mail.mil

\begin{abstract}
Super-aligned carbon nanotube (CNT) thin films offer the capability to be placed into compact lightweight sound sources for active noise control, loudspeaker, or other acoustic applications. The superaligned CNT films generate thermoacoustically induced oscillations to generate sound. This occurs through the use of a dynamic signal, such as applying an alternating current in the audio frequency range to the thin film. This mechanism is carried out through joule heating which heats the air above the surface, thereby producing sound waves from periodic air vibration. Here, we report an investigation of the sound pressure and thermal properties of advanced carbon nanotube thin films with an emphasis to address the signal distortion issue.
\end{abstract}

Index Terms - Thermophone, Carbon Nanotube thin film loudspeaker.

\section{INTRODUCTION}

Acoustic phenomena can be observed when conductors are electrically heated with alternating current when either free-standing or supported by a substrate. Materials with small heat capacity per unit area posses outstanding thermoacoustic properties and can be used in thermally driven loudspeakers such as thermophones. In 1917, Arnold and Crandall developed the theory of the thermophone and described the behavior such that as current is passed through thin conducting materials a periodic heating sets up temperature waves which are propagated into the surrounding medium. The thermal contraction and expansion of the surrounding medium determine the amplitude of the resulting sound waves [1]. The alternate expansion and contraction of the boundary layer of air in the immediate vicinity of the thin film causes a pressure otherwise known as a sound wave. It is important to note that the sound is produced from the thermal expansion and contraction of the air in the vicinity of the thin film, and not from the mechanical movement of the thin film. However at acoustic frequencies these waves are attenuated to a small fraction of their original amplitude and at under certain operating power we observe that distortion becomes a dominate feature [2]. Other studies have recommended to add a dc offset voltage or a pulse width modulated signal to eliminate the doubling of frequency [3]. The focus of this paper is to verify their recommendation.

\section{EXPERIMENTAL DETAILS \\ 1. Synthesis}

The thin films are comprised of multiwall carbon nanotubes (MWCNTs) directly drawn from superaligned CNT forests that are grown on quartz substrates by chemical vapor deposition methods. The lengths of the CNTs are $500-700 \mu \mathrm{m}$ and the diameters range from $30-50 \mathrm{~nm}$. Hitachi S-4700 scanning electron microscope analysis was performed and a representative forest is shown in Figure 1. The strong van der Waals interactions among neighboring CNTs allow for the CNTs to be attached end to end such that the thin film can be directly pulled from the array.

\section{Sample Preparation and Thermal Characterization}

The CNT film was directly pulled across two copper electrodes that serve as the points of contact for the ground and current flow across the sample as shown in Figure 2. A small amount of conducting silver electrode paint (DuPont CB028 Silver Paint) was applied to the poles prior to the deposition of the thin film. The silver paint serves as an adhesive for the thin film but also improves the electrical contact between the CNT film and the copper electrode. The heat capacity and in-plane thermal conductivity of the super-aligned CNTs were determined by digital scanning calorimetry (DSC) (Perkin Elmer). The average heat capacity of the superaligned CNT film is $490 \mathrm{~kJ} \mathrm{~kg}^{-1} \mathrm{~K}^{-1}$ and the thermal conductivity is $0.23 \mathrm{~W} / \mathrm{mK}$.

\section{RESULTS AND DISCUSSION}

The CNT thin film produces a pressure oscillation in the surrounding air as it is periodically heated and cooled. This pressure oscillation leads to the generation of thermoacoustic sound over a wide frequency range.

DISTRIBUTION A: Approved for public release; distribution is unlimited. 
The experimental measurements follow a trend where the sound pressures level-off with increasing frequencies; this trend is demonstrated as a transition from the far field to the near field sound propagation as

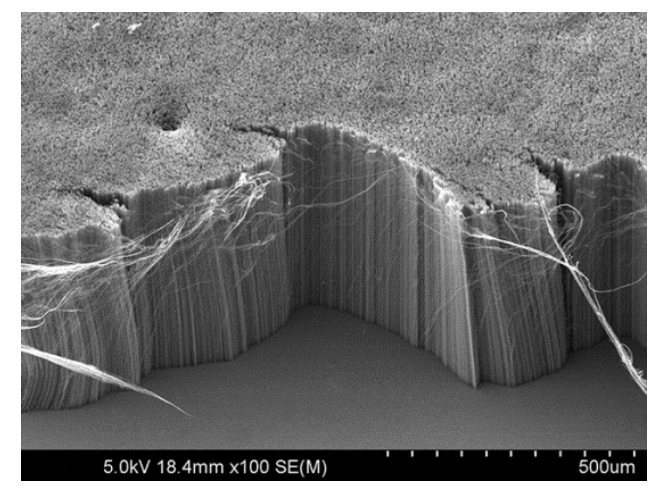

Fig. 1 SEM image of as-grown super aligned CNT arrays.

the frequency is increased. The measurements were performed in an anechoic chamber with a pure sinusoidal voltage applied to a free standing CNT thin film positioned $5 \mathrm{~cm}$ from a microphone. The output of the free-standing film reaches $90 \mathrm{~dB}$ of sound pressure at 30 $\mathrm{W}$ of power, as illustrated in Figure 3. The thermal excitation was acquired with an MIKRON TS7300 IR camera. At a power of $45 \mathrm{~W}$ and an acoustic output of 90 $\mathrm{dB}$, the maximum temperature across the surface of the free standing thin film peaked at $320^{\circ} \mathrm{F}$ as depicted in Figure 3.

The audio frequency produced was twice the frequency of the $\mathrm{AC}$ current applied to the thin film speaker. This signal distortion is due to the power dissipated as being proportional to the square of the input current. In the experiment, a squared alternating current I sin $(\omega t)$ was supplied, wherein the Joule heating is proportional to

$$
R I^{2} \sin ^{2}(\omega t)=\frac{R I^{2}}{2}(1-\cos (2 \omega t))(1)
$$

the acoustic frequency is given by $\omega=2 \pi f$. Experimental measurements confirmed that the squared sinusoidal input caused a frequency doubling in the sound pressure waveform.

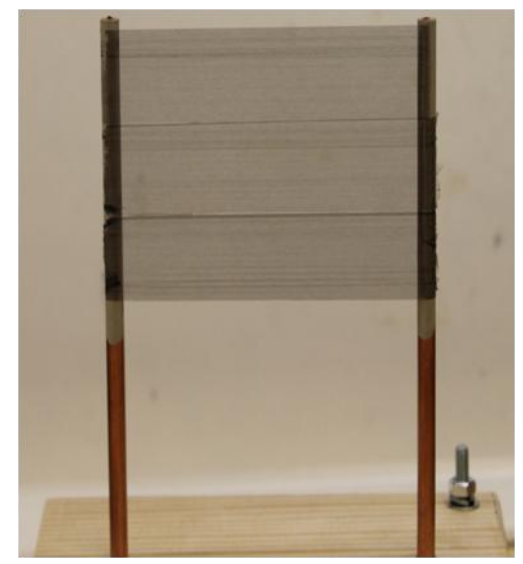

Fig. 2 A super-aligned CNT film placed across two copper electrodes

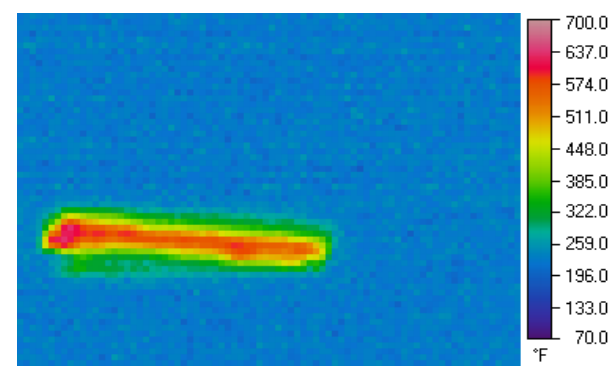

Fig. $3 \mathrm{CNT}$ free standing thin film peaked at $320^{\circ} \mathrm{F}$

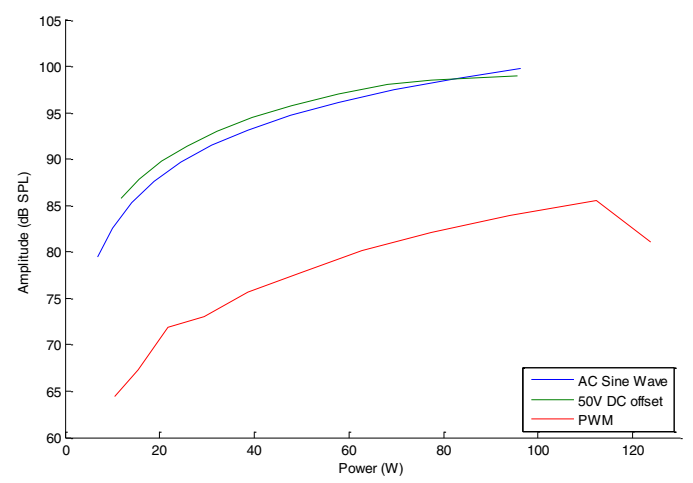

Fig. 4 Output power as a function of sound pressure of super-aligned CNT film with an applied AC sine wave, 50V DC offset, and PWM signal.

Experimental test were conducted with an AC sine wave, $50 \mathrm{~V}$ dc offset, and PWM signal with an applied voltage ranging from $100-300 \mathrm{~V}$ to a free-standing CNT thin film. An input frequency of $2 \mathrm{kHz}$ signal with energy of the dominate tones at $2 \mathrm{kHz}$ and $4 \mathrm{kHz}$ for all three scenarios.

As shown in Figure 4, the sound output of the CNT array in decibel (dB) sound pressure level (SPL) is plotted as a function of the applied input voltage and the type of input signal. For consistency purposes, the same CNT loudspeaker specimen was tested with 3 different types of signal applications: (1)AC sine wave, (2) AC sine wave with $50 \mathrm{~V}$ DC offset, and (3) pulse width modulation (PWM). The 50V DC offset test resulted in a two to three $\mathrm{dB}$ increase as compared to the same sample tested without a 50V DC shift. A DC offset was applied with the intentions to shift the signal reference from $0 \mathrm{~V}$ to half the peak-to-peak value. As a result the signal only has one peak current in one cycle, as shown in Figure 5.

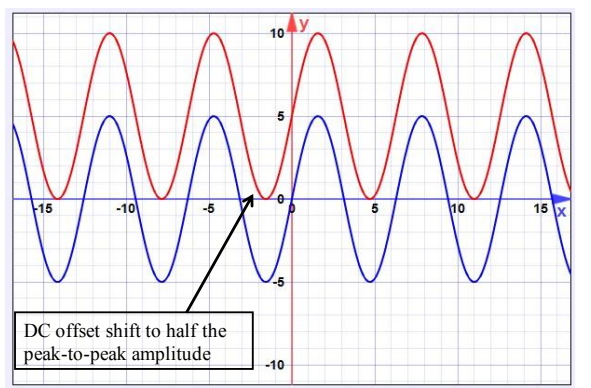

Fig. 5 Blue signal has two peaks at $+5 \mathrm{~V}$ and $-5 \mathrm{~V}$, whereas the red signal has only one peak at $10 \mathrm{~V}$

DISTRIBUTION A: Approved for public release; distribution is unlimited. 


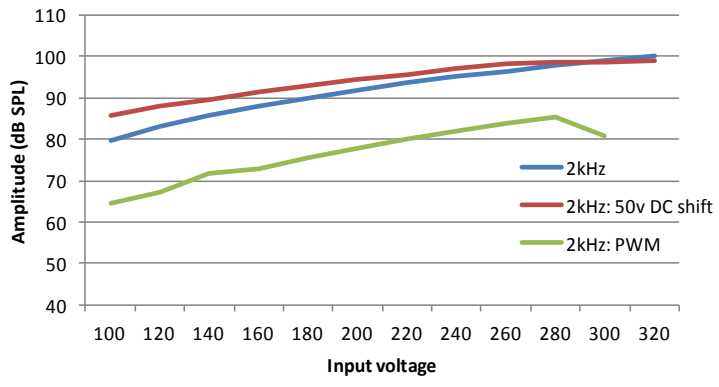

Fig. 6 Sound output as a function of input voltage and output SPL

Figure 6 displays the sound output as a function of input voltage and output SPL. For the PWM test, we started at $100 \mathrm{~V}_{\text {pk-pk }}$ with $50 \%$ duty cycle. As a result the CNT loudspeaker displayed a noticeable drop in the SPL. With a PWM signal it is automatically on and off as compared to the gradual on-and-off sine wave. The rate of switching may not give an appropriate response time for the CNT. In PWM signal, current reaches its maximum peak instantaneously, whereas in sine wave there is continuous variation in both amplitude and direction, which would give us the oscillatory heating and molecular vibration needed to create the pressure/sound wave from the CNTs. Furthermore, the dip in sound level at $300 \mathrm{~V}$ for the PWM signal was due to sparking and subsequent destruction of part of the CNT array. PWM is modulation of the average amplitude of the output signal by adjusting the width of the on and off time of the signal.

Distortion is interpreted as the growth in harmonics and energy exciting the vibrating modes at the same rate as the dominate harmonic. Figure 7 displays the total harmonic distortion plus noise $(\mathrm{THD}+\mathrm{N})$ plotted as a function of the applied input voltage and input signal. For the $2 \mathrm{kHz}$ signal with no DC offset, the band reject filter was set to the predicted $4 \mathrm{kHz}$ for the purposes of the measurement. For the $2 \mathrm{kHz}$ input with a $50 \mathrm{~V} \mathrm{DC}$ offset, the band reject filters were centred on the predicted 2 and $4 \mathrm{kHz}$. The PWM signal is not plotted since there is energy at each successive harmonic of the input, thus a measure of $\mathrm{THD}+\mathrm{N}$ would be difficult to interpret.

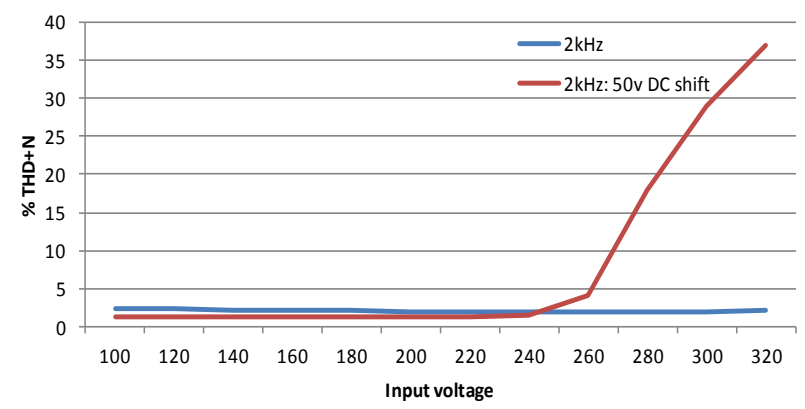

Fig. 7 Sound output as a function of input voltage and $\%$ total harmonic distortion plus noise

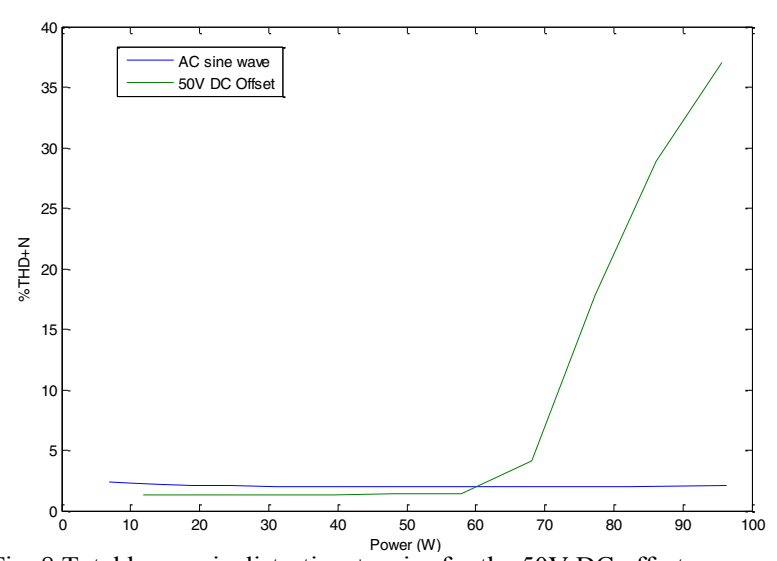

Fig. 8 Total harmonic distortion + noise for the $50 \mathrm{~V}$ DC offset

Figure 8 shows the total harmonic distortion plus noise for the $50 \mathrm{~V}$ DC offset test as function of power. For the DC offset test, the trend shows that up to a certain point it is linear, and then at $260 \mathrm{~V}$ it hits a critical point where the vibrating mode is responding at the same rate at the dominating harmonic tone as shown in Figure 7. Once the output power surpasses a critical point, it is suspected that the air molecules of the boundary layer between the free standing CNT thin film and surrounding air are vibrating at such a rate that each successive harmonic has equivalent energy. Figure 9 shows that with a DC offset the distortion actually increases.

As the power increases, there is a linear increase in the energy of the dominate tones as well as the resulting harmonics. Once a critical power input is reached there is a non-linear increase in harmonic distortion (i.e. the ratio of energy between dominant tones and harmonics).

Figure 9 depicts the Fast Fourier Transform (FFT) of output frequency response (successive harmonics) as a function of amplitude for the AC applied signal, 50V DC

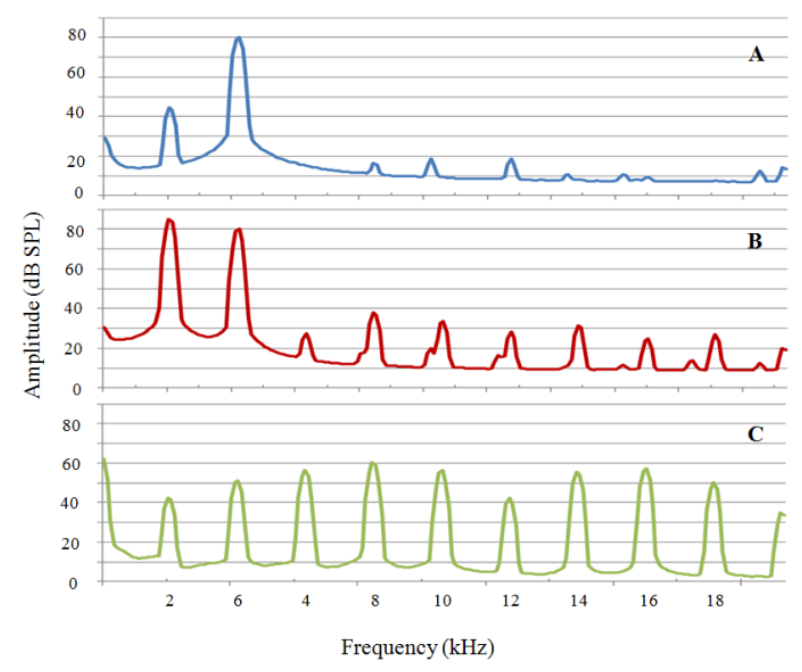

Fig. 9 Fast Fourier Transform (FFT) of output for a $2 \mathrm{kHz}$ input tone

DISTRIBUTION A: Approved for public release; distribution is unlimited. 
offset, and PWM test. Panel A shows that the energy is primarily at twice input frequency $(2 \mathrm{~F}=4 \mathrm{kHz})$, as the applied $2 \mathrm{kHz}$ input frequency with no apparent distortion in the successive harmonics. Panel B depicts FFT output for a $2 \mathrm{kHz}$ tone and that there is now significant energy at the input frequency and $2 \mathrm{~F}$ for the $50 \mathrm{~V}$ DC offset test run. Panel $\mathrm{C}$ displays a FFT output for a $2 \mathrm{kHz}$ tone with a PWM applied. Note that there is significant energy at successive harmonics of the input frequency.

\section{CONCLUSIONS}

The goal of this study was to mitigate signal distortion by applying a pulse-width modulation, or a DC offset. Unexpectedly, our studies have shown that PWM increases energy and produces an increase in the energy of the dominant tones and harmonics. While a DC offset can be added to the system, once it reaches a critical power, the vibrating mode is responding at the same rate as the dominating harmonic tone. More than 10 CNT thermoacoustic thin films have been fabricated and tested. The acoustic outputs consistently reached over $80 \mathrm{~dB}$. At a critical power threshold of $57 \mathrm{~W}$ a significant amount of distortion occurred for both instances involving either a DC offset or PWM signal. Future work will include gaining a deeper understanding of the distortion effects as a function of temperature, or even possible direction of air flow or trapped air surrounding the thin film.

\section{REFERENCES}

[1] Arnold and Crandall, "Thermophone as a Precision Source of Sound", Phys. Rev. 10 [1] (1917) 22-38

[2] Wente, "The Thermophone", Phys Rev. 19 [4] (1922), 333-345,

[3] Aliev et al. "Thermal Transport in MWCNT sheets and yarn, Carbon 45(2007) 2880-2888.

[4] Baloch et al. "Remote Joule heating by a carbon nanotube", Nature Nanotechnology, 39, (2012),1-4

[5] Jiang et al. "Superaligned Carbon Nanotube Arrays, Films, and Yarns: A Road to Applications", Advanced Materials, 23, (2011) 1154-1161

[6] Tian et al. "Graphene-on-Paper Sound Source Devices", American Chemical Society (2011)

[7] Xiao et al. "Flexible, Stretchable, Transparent Carbon Nanotube Thin Film Loudspeakers", Nano Letters, 8[12] ( 2008) 